\title{
Modeling of Nucleon-Nucleon Potentials, Quantum Inversion versus Meson Exchange Pictures*
}

\author{
L. Jäde, M. Sander and H. V. von Geramb \\ Theoretische Kernphysik, Universität Hamburg \\ Luruper Chaussee 149, D-22761 Hamburg
}

(October 10, 2018)

\begin{abstract}
The notion of interacting elementary particles for low and medium energy nuclear physics is associated with definitions of potential operators which, inserted into a Lippmann-Schwinger equation, yield the scattering phase shifts and observables. In principle, this potential carries the rich substructure consisting of quarks and gluons and thus may be deduced from some microscopic model. In this spirit we propose a boson exchange potential from a nonlinear quantum field theory. Essentially, the meson propagators and form factors of conventional models are replaced by amplitudes derived from the dynamics of self-interacting mesons in terms of solitary fields. Contrary to deduction, we position the inversion approach. Using Gel'fand-Levitan and Marchenko inversion we compute local, energy-independent potentials from experimental phase shifts for various partial waves. Both potential models give excellent results for on-shell NN scattering data. In the off-shell domain we study both potential models in $(p, p \gamma)$ Bremsstrahlung, elastic nucleon-nucleus scattering and triton binding energy calculations. It remains surprising that for
\end{abstract}

*Contribution to the International Conference on Inverse and Algebraic Quantum Scattering Theory, Lake Balaton '96. 
all observables the inversion and microscopic meson exchange potentials are equivalent in their reproduction of data. Finally, we look for another realm of elementary interactions where inversion and meson exchange models can be applied with the hope to find more sensitivity to discern substructure dynamics.

\section{INTRODUCTION}

In the last decade, the development of highly quantitative models for the nucleon-nucleon interaction was one of the major tasks of theoretical nuclear physicists. Based on Yukawa's pioneer work, potentials for NN forces mediated by the exchange of boson fields with masses below $1 \mathrm{GeV}$ were invented and successfully applied to various problems in medium energy nuclear physics [1]. Despite of their remarkable ability to account for quantitative details of NN phenomenology, none of these models contains any reference to Quantum Chromodynamics QCD, which is believed to be the underlying microscopic theory of the strong interaction. There are a number of models which explicitly refer to QCD [2], but so far all of them fail to describe the nucleon-nucleon interaction comparable well as the phenomenological boson exchange potentials. Thus the major shortcomings of today's nucleon-nucleon potential models are the empirical character of the boson-exchange potentials which arises from phenomenological usage of form factors without stringent connections to QCD and on

the other side the failure of QCD inspired models to provide a quantitative description of nucleon-nucleon scattering data.

The goal of the Solitary Boson Exchange Potential, which will be described in detail in section [II] is to interpolate between these extreme positions [3]. Characteristic features of QCD inspired potential models, namely their nonlinear character, are taken into account using a nonlinear expansion of the Klein-Gordon equation as equation of motion for the boson fields. On the other hand, the solutions of this equation, called solitary boson fields, are used in a boson exchange potential which is in great analogy to the Bonn-B potential [4] to 
obtain a quantitative description of NN data comparable well as the phenomenological boson exchange potentials. It will turn out that the nonlinear character of the boson fields allows to substitute the phenomenological form factors of the Bonn potential. Simultaneously, an empirical scaling law is found which relates all meson parameters, as expected in a model based on QCD.

Contrary to the microscopic models, potentials obtained from quantum inversion were developed for various hadron-hadron interactions [5]. Using experimental phase shifts as input in the Gel'fand-Levitan and Marchenko inversion algorithm for the Sturm-Liouville equation yields local potential operators in coordinate space which are model-independent and reproduce the experimental data by construction.

As far as elastic NN data are concerned, inversion potentials evidently provide a precise description of the NN interaction. It is a nontrivial question, however, whether this accuracy remains in the application of inversion potentials to more complex problems. Since the scattering phase shifts, from which inversion potentials are obtained, only contain the onshell information of the scattering amplitude, i.e. the absolute value of the incoming and outgoing nucleon momentum remains unchanged, it is questionable if such a potential can account for the description of reactions where the off-shell part of the $t$-matrix contributes. A sensitivity on details of the off-shell amplitude would provide the desired possibility to test possible effects of the substructure of potential models. In particular, we seek a signature in the data to confirm the assumptions which led to the Solitary-Boson-Exchange-Potential. To study this interesting point we apply boson exchange as well as inversion potentials to calculate the differential cross section for $(p, p \gamma)$ Bremsstrahlung and elastic nucleonnucleus scattering as well as the triton binding energy. The astonishing outcome implies that inversion and boson exchange potentials yield equivalent results. Even more surprising, an improvement of the description of the on-shell data enhances the accuracy describing the off-shell data.

Before inversion and boson exchange potentials will be compared, we give a short reminder of the algorithms which are used to calculate inversion potentials from experimental 
phase shifts and show the typical structure of boson exchange potentials outlining the basic ideas of our Solitary-Boson-Exchange-Potential model.

\section{NUCLEON-NUCLEON POTENTIALS FROM INVERSION}

Contrary to the direct path to obtain a potential for NN scattering from some microscopic model, the algorithm of quantum inversion can be applied using experimental phase shifts as input [5]. Nowadays, the inversion techniques for nucleon-nucleon quantum scattering have evolved up to almost perfection for scattering data below pion production threshold. Numerically, input phase shifts can be reproduced for single and coupled channels with a precision of $1 / 100$ of a degree, which is much lower than the experimental uncertainty. This accuracy and the possibility to test the inversion potential 'online', i. e. inserting the potential into the scattering equation to reproduce the input phase shifts, makes them a reliable and easy-to-handle tool for highly quantitative medium energy nuclear physics. Guided by this spirit, the utmost aim of quantum inversion is to provide the most simple operator to reproduce the scattering data. This paradigm, however, proscribes to include

any momentum dependence and thus any non-locality in the potential since this would open a box full of ambiguities which can not be associated with the goal of simplicity.

As a basis, the radial Schrödinger equation is assumed to be the relevant equation of motion for the two-particle system

$$
\left\{-\frac{d^{2}}{d r^{2}}+\frac{\ell(\ell+1)}{r^{2}}+\frac{2 \mu}{\hbar^{2}} V_{\ell}(r)\right\} \psi_{\ell}(k, r)=k^{2} \psi_{\ell}(k, r),
$$

where $V_{\ell}(r)$ is a local, energy-independent operator in coordinate space. Substituting

$$
q(r)=\frac{\ell(\ell+1)}{r^{2}}+\frac{2 \mu}{\hbar^{2}} V_{\ell}(r) \quad \text { and } \quad \lambda=k^{2},
$$

one obtains the well-known Sturm-Liouville equation

$$
\left[-\frac{d^{2}}{d x^{2}}+q(x)\right] y(x)=\lambda y(x) .
$$


The scattering phase shifts enter as boundary conditions for the physical solutions of (2.1) which read

$$
\lim _{r \rightarrow \infty} \psi_{\ell}(k, r)=\exp \left(i \delta_{\ell}(k)\right) \sin \left(k r-\frac{\ell \pi}{2}+\delta_{\ell}(k)\right)
$$

There are two equivalent inversion algorithms for the Sturm-Liouville equation, the Marchenko and the Gel'fand-Levitan inversion, which will be outlined in the next sections for the case of uncoupled channels.

\section{A. Marchenko Inversion}

In the Marchenko inversion the experimental information enters via the $S$-matrix, which is related to the scattering phase shifts by the simple relation

$$
S_{\ell}(k)=\exp \left(2 i \delta_{\ell}(k)\right)
$$

Inserting a rational representation of the $S$-matrix [5] into the integral equation for the input kernel

$$
F_{\ell}(r, t)=-\frac{1}{2 \pi} \int_{-\infty}^{+\infty} h_{\ell}^{+}(k r)\left[S_{\ell}(k)-1\right] h_{\ell}^{+}(k t) d k
$$

where $h_{\ell}^{+}(x)$ are the Riccati-Hankel functions, the Marchenko equation

$$
A_{\ell}(r, t)+F_{\ell}(r, t)+\int_{r}^{\infty} A_{\ell}(r, s) F_{\ell}(s, t) d s=0
$$

becomes an algebraic equation for the translation kernel $A_{\ell}(r, t)$. The potential is obtained by taking the derivative

$$
V_{\ell}(r)=-2 \frac{d}{d r} A_{\ell}(r, r)
$$

\section{B. Gel'fand-Levitan Inversion}

Instead of the $S$-matrix, in the Gel'fand-Levitan inversion the Jost-matrix carries the experimental input. The latter is related to the $S$-matrix by 


$$
S_{\ell}(k)=\frac{F_{\ell}(-k)}{F_{\ell}(k)}
$$

and leads to the input kernel

$$
G_{\ell}(r, t)=\frac{2}{\pi} \int_{0}^{\infty} j_{\ell}(k r)\left[\frac{1}{\left|F_{\ell}(k)\right|^{2}}-1\right] j_{\ell}(k t) d k
$$

where $j_{\ell}(x)$ are the Riccati-Bessel functions. A rational representation of the spectral density [5] again yields an algebraic form for the Gel'fand-Levitan equation

$$
K_{\ell}(r, t)+G_{\ell}(r, t)+\int_{0}^{r} K_{\ell}(r, s) G_{\ell}(s, t) d s=0,
$$

and the desired potential is obtained from

$$
V_{\ell}(r)=2 \frac{d}{d r} K_{\ell}(r, r)
$$

\section{Coupled Channel Inversion}

For coupled channels, i. e. transitions between states with different angular momentum $\ell_{i}$, the Schrödinger equation (2.1) becomes a matrix equation.

$$
\left\{-\frac{d^{2}}{d r^{2}} \mathbf{1}+\mathbf{V}(r)\right\} \boldsymbol{\Psi}(r)=k^{2} \boldsymbol{\Psi}(r)
$$

where in the case of two coupled angular momentum the potential matrix reads

$$
\mathbf{V}(r)=\left(\begin{array}{cc}
\frac{\ell_{1}\left(\ell_{1}+1\right)}{r^{2}}+\frac{2 \mu}{\hbar^{2}} V_{\ell_{1} \ell_{1}}(r) & \frac{2 \mu}{\hbar^{2}} V_{\ell_{1} \ell_{2}}(r) \\
\frac{2 \mu}{\hbar^{2}} V_{\ell_{2} \ell_{1}}(r) & \frac{\ell_{2}\left(\ell_{2}+1\right)}{r^{2}}+\frac{2 \mu}{\hbar^{2}} V_{\ell_{2} \ell_{2}}(r)
\end{array}\right)
$$

The input and translation kernels of the previous sections and in particular the $S$-matrix now generalize to matrices. Since it is cumbersome for coupled channel situations to solve the Riemann-Hilbert Problem (2.9) numerically we will focus on the Marchenko inversion which

does not include any serious difficulty for the general case of coupled channels. Defining the 
diagonal matrix which contains the Riccati-Hankel functions by

$$
\mathbf{H}(x)=\left(\begin{array}{cc}
h_{\ell_{1}}^{+}(x) & 0 \\
0 & h_{\ell_{2}}^{+}(x)
\end{array}\right),
$$

one gets as a generalization of (2.6) for the input kernel

$$
\mathbf{F}(r, t)=-\frac{1}{2 \pi} \int_{-\infty}^{+\infty} \mathbf{H}(k r)[\mathbf{S}(k)-\mathbf{1}] \mathbf{H}(k t) d k+\sum_{i=1}^{N_{B}} \mathbf{H}\left(k_{i} r\right) \mathbf{N}\left(k_{i}\right) \mathbf{H}\left(k_{i} t\right),
$$

where the matrix $\mathbf{N}\left(k_{i}\right)$ contains the asymptotic normalizations of the wave functions for the bound states at (imaginary) momentum $k_{i}$. The Marchenko fundamental equation (2.7) now reads

$$
\mathbf{A}(r, t)+\mathbf{F}(r, t)+\int_{r}^{\infty} \mathbf{A}(r, s) \mathbf{F}(s, t) d s=0
$$

and the potential matrix is obtained from

$$
\mathbf{V}(r)=-2 \frac{d}{d r} \mathbf{A}(r, r)
$$

\section{THE ONE-SOLITARY-BOSON-EXCHANGE POTENTIAL}

In the following section, our Solitary-Meson-Exchange-Potential model is used to demonstrate the major problems in the modeling of nucleon-nucleon potentials using the boson exchange picture. As mentioned in the introduction, today's models for NN interaction either refer to QCD but can not describe scattering data or - as is the case for the conventional boson exchange potentials - they contain empirical entities which must be fitted to experiment.

The One-Solitary-Boson-Exchange-Potential OSBEP which was recently developed by the Hamburg group [3] tries to interpolate between these extreme positions. Surprisingly, it turns out that the inclusion of typical features from QCD inspired models can account for the empirical parts of usual boson exchange potentials preserving the high accuracy 
in the description of data. Therefore, we take the OSBEP as an illustrative example of how a microscopic model is used to obtain a boson exchange potential for nucleon-nucleon interactions.

\section{A. Solitary Mesons}

Motivated by the nonlinear character of QCD, we assume a nonlinear self-interaction for all mesons which enter the boson exchange potential. Doing so, we use the model of solitary mesons developed by Burt [6]. Here the decoupled meson field equation is parameterized by

$$
\partial_{\mu} \partial^{\mu} \Phi+m^{2} \Phi+\lambda_{1} \Phi^{2 p+1}+\lambda_{2} \Phi^{4 p+1}=0
$$

where $\Phi$ is the operator to describe the self-interacting fields. For mesons with nonzero spin this operator is a vector in Minkowski space. The parameter $p$ equals $1 / 2$ or 1 to yield odd or even powered nonlinearities. Using this parameterization, equation (3.1) can be solved analytically. The solutions are represented as a power series in $\varphi$ [6]

$$
\Phi=\sum_{n=0}^{\infty} C_{n}^{1 / 2 p}(w) b^{n} \varphi^{2 p n+1},
$$

where $\varphi$ is a solution of the free Klein-Gordon equation with meson mass $m$. These special wavelike solutions of (3.1) are oscillating functions which propagate with constant shape and velocity. Corresponding to the classical theory of nonlinear waves they shall be called solitary meson fields. The coefficients $C_{n}^{a}(w)$ are Gegenbauer Polynomials, $b$ and $w$ are functions of the coupling constants and the order $p$ of the self-interaction. To quantize the solitary fields we use free wave solutions of the Klein-Gordon equation in a finite volume $V$ |7]

$$
\varphi(x, k):=\frac{1}{\sqrt{2 D_{k} \omega_{k} V}} a(k) e^{-i k x},
$$

where the operator $a(k)$ annihilates and the hermitian adjoint $a^{\dagger}(k)$ creates quanta of positive energy

$$
\omega_{k}^{2}=\vec{k}^{2}+m^{2}
$$


At this point it is important to notice that we added a factor $D_{k}$ which is an arbitrary Lorentz invariant function of $\omega_{k}$. As will become obvious later, this constant is crucial for the proper normalization of solitary waves.

The probability for the propagation of an interacting field can now be defined as the amplitude to create an interacting system at some space-time point $x$ which is annihilated into the vacuum at $y$. Since the intermediate state is not observable and the particles are not distinguishable a weighted sum over all intermediate states has to be performed [8]

$$
\begin{aligned}
& i P(y-x)= \\
& \sum_{k} \sum_{N=0}^{\infty} \frac{1}{N !}\left[\left\langle 0|\Phi(y, k)| N_{k}\right\rangle\left\langle N_{k}\left|\Phi^{\dagger}(x, k)\right| 0\right\rangle \theta\left(y_{0}-x_{0}\right)\right. \\
& \left.+\left\langle 0|\Phi(x, k)| N_{k}\right\rangle\left\langle N_{k}\left|\Phi^{\dagger}(y, k)\right| 0\right\rangle \theta\left(x_{0}-y_{0}\right)\right] .
\end{aligned}
$$

A straight forward calculation yields the desired amplitude in momentum space

$$
\begin{aligned}
& i P\left(k^{2}, m\right)=\sum_{n=0}^{\infty}\left[C_{n}^{1 / 2 p}(w)\right]^{2} \\
& \times \frac{b^{2 n}}{(2 V)^{2 p n}} \frac{(2 p n+1)^{2 p n-2}}{D_{k}^{2 p n+1}\left(\vec{k}^{2}+M_{n}^{2}\right)^{p n}} i \Delta_{F}\left(k^{2}, M_{n}\right),
\end{aligned}
$$

with the Feynman propagator

$$
i \Delta_{F}\left(k^{2}, M_{n}\right)=\frac{i}{k_{\mu} k^{\mu}-M_{n}^{2}}
$$

and a mass-spectrum

$$
M_{n}=(2 p n+1) m .
$$

Since $V \cdot \omega_{k}$ is a Lorentz-scalar the amplitude (3.5) is Lorentz invariant. At this point it is convenient to introduce the dimensionless coupling constants $\alpha, \alpha_{1}$ and $\alpha_{2}$ which we define as

$$
\begin{aligned}
\alpha & :=\frac{b}{(2 m V)^{p}}, \\
\alpha_{1} & :=\frac{\lambda_{1}}{4(p+1) m^{2}(2 m V)^{p}},
\end{aligned}
$$




$$
\alpha_{2}:=\frac{\lambda_{2}}{4(2 p+1) m^{2}(2 m V)^{2 p}}
$$

This yields

$$
w=\frac{\alpha_{1}}{\sqrt{\alpha_{1}^{2}-\alpha_{2}}},
$$

and

$$
\begin{aligned}
& i P\left(k^{2}, m\right)=\sum_{n=0}^{\infty}\left[C_{n}^{1 / 2 p}(w)\right]^{2} \\
& \times \frac{\left[\left(m^{p} \alpha_{1}\right)^{2}-m^{2 p} \alpha_{2}\right]^{n}(2 p n+1)^{2 p n-2}}{D_{k}^{2 p n+1}\left(\vec{k}^{2}+M_{n}^{2}\right)^{p n}} i \Delta_{F}\left(k^{2}, M_{n}\right) .
\end{aligned}
$$

The amplitude (3.9) shall be referred to as modified solitary meson propagator. For $p=1$ one gets the amplitude for the propagation of pseudoscalar fields, $p=1 / 2$ describes scalar particles. The series (3.9) converges rapidly, depending on the mass the subsequent terms diminish by two $(\pi)$ or three $(\omega)$ orders of magnitude and in practical calculations it is sufficient to use $n_{\max }=4$.

\section{B. Proper Normalization}

The propagator (3.9) contains the arbitrary constant $D_{k}$ which can depend on the energy $\omega_{k}$ and the coupling constants and is fixed by the conditions [6]

- all amplitudes must be Lorentz invariant,

- $D_{k}$ must be dimensionless,

- all self-scattering diagrams must be finite,

- the fields have to vanish for $\lambda_{1}, \lambda_{2} \rightarrow 0$.

Whereas the first three conditions are evident the last one requires to recall: 
If a particle has no interaction then there is no way to create or measure it and the amplitude for such a process vanishes. The field exists solely because of its interaction.

The proper normalization constant is a powerful tool to avoid the problem of regularization which arises in conventional models. In a $\lambda \Phi^{4}$-theory for example, which is described by setting $\lambda_{2}=0$ and $p=1$, one gets infinite results calculating the first correction to the two-point function $i P\left(k^{2}, m\right)$. A proper normalization, i.e. using the smallest power

$$
D_{k} \sim\left(\omega_{k} V\right)^{2}
$$

makes the result finite. A different situation occurs in models including massive spin1 bosons. Such a case, with or without self-interaction, is harder to regularize due to the additional momentum dependence which arises from the tensor structure in Minkowski space. Nevertheless, the vector mesons $\rho$ and $\omega$ are important ingredients in every boson exchange model. A minimum power proper normalization to solve this problem is

$$
D_{k} \sim\left(\omega_{k} V\right)^{4}
$$

In summary, we satisfy the above stated four conditions with

$$
\begin{aligned}
& D_{k}=\left\{1+\left[\left(\frac{m^{2}}{\lambda_{1}}\right)^{\frac{2}{p}}+\left(\frac{m^{2}}{\lambda_{2}}\right)^{\frac{1}{p}}\right]\left(\omega_{k} V\right)^{2}\right\}^{\kappa} \\
& \text { where: } \begin{cases}\kappa=1 & \text { for scalar and } \\
& \text { pseudoscalar mesons. } \\
\kappa=2 & \text { for vector mesons. }\end{cases}
\end{aligned}
$$

\section{The Benefits of OSBEP}

With its proper normalization, the solitary meson propagator now is completely determined and can be applied in a boson exchange potential. In conventional models a 
renormalized Feynman propagator is used to describe the meson propagation. Additionally, an empirical form factor is attached to each vertex to achieve convergence in the scattering equation. In a model with solitary mesons as excess particles the solitary meson propagator (3.5) should be used instead of the Feynman propagator. Due to the proper normalization, the solitary meson propagator already carries a strong decay with increasing momentum and thus offers the possibility to forgo the form factors. Therefore, proper normalization not only cures the problem to regularize the meson self-energy but simultaneously provides a meson propagator which is able to substitute the form factors.

Additionally, comparing the properly normalized solitary meson propagators to the Bonn-B form factors, we find an empirical scaling relation for the meson self-interaction coupling constants (3.7). We regarded the simple case $\lambda_{2}=0$ to obtain [3]

$$
\alpha(m)=\alpha_{\pi} \cdot\left(\frac{m_{\pi}}{m}\right)^{\frac{1}{2}} \quad \text { for scalar fields }
$$

and: $\quad \frac{\alpha(m)}{\sqrt{\kappa}}=\frac{\alpha_{\pi}}{\sqrt{\kappa_{\pi}}} \cdot\left(\frac{m_{\pi}}{m}\right) \quad$ for pseudoscalar

and vector fields.

Thus, the pion self-interaction coupling constant $\alpha_{\pi}$ is the only parameter to describe the meson dynamics. This connection between masses and coupling constants can be anticipated in a model which is motivated by QCD and thus puts physical significance into the parameter $\alpha_{\pi}$.

Together with the meson-nucleon coupling constants the model contains a total number

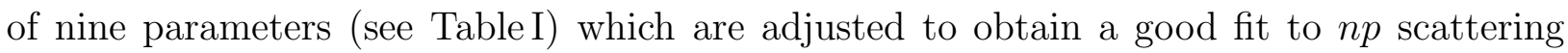
phase shifts and deuteron data (see Table $\amalg$ and Figure2). The agreement with scattering observables (Figure囵) is excellent and the goal to achieve a description of experimental data which is comparable to the Bonn-B potential is accomplished. 


\section{THE QUEST FOR OFF-SHELL EFFECTS}

Concerning nucleon-nucleon scattering, boson exchange models and inversion potentials show excellent agreement with experimental data. The results of the OSBEP and BonnB models are shown in Figure2, the inversion potentials reproduce the phase shifts by construction. The next step is to apply both potential models in more complex reactions to find out whether the absent off-shell information in the inversion potentials would lead to a break-down of the model in situations where the off-shell part of the scattering amplitude contributes. Three of such processes have been considered and shall be reviewed here: Application of model potentials in $(p, p \gamma)$ Bremsstrahlung [9], calculations of triton binding energy [10] and - most recently - usage of boson exchange and inversion potentials in an in-medium, full-folding optical potential model for nucleon-nucleus scattering [11].

The strategy to compare boson exchange and inversion potentials is as follows. We take some model potential $t$-matrix $t_{\text {mod }}\left(k, k^{\prime}\right)$ with its characteristic off-shell (i.e. $k \neq k^{\prime}$ ) behavior, calculate the phase shifts from the on-shell part $t_{\text {mod }}(k, k)$, use these model phase shifts to calculate an inversion potential $V_{\ell}(r)$, as described in Section!l, which in turn yields a $t$-matrix $t_{i n v}\left(k, k^{\prime}\right)$ where the off-shell behavior is uniquely defined by the restriction [5

$$
\int_{a}^{\infty} r\left|V_{\ell}(r)\right| d r<\infty \quad \text { for } \quad a \geq 0 .
$$

Therefore, any on-shell difference of inversion potentials will lead to off-shell differences in the $t$-matrix. On the other hand, the off-shell behavior of the model $t$-matrix and the $t$-matrix obtained from inversion will in general be entirely different, at least for large

momenta. Therefore, comparing $t_{m o d}\left(k, k^{\prime}\right)$ and $t_{i n v}\left(k, k^{\prime}\right)$ in off-shell sensitive reactions works as a tool to test whether off-shell differences of on-shell equivalent $t$-matrices manifest themselves in the calculation of observable data. 


\section{A. Cross Sections for $(p, p \gamma)$ Bremsstrahlung}

In Figure 3, the theoretical $(p, p \gamma)$ cross sections for several potentials are shown. In detail, Jetter et al. [9] calculated cross sections using the boson exchange potentials Paris and Bonn-B [1] as well as inversion potentials from the Nijmegen PWA [12] and phase shifts from the Bonn-B potential. Two kind of calculations for $(p, p \gamma)$ cross sections were applied. First, an on-shell approximation which is shown as upper curve in Figure 3 and clearly misses the data, second an exact off-shell calculation which is represented by the lower curve. Obviously, using the exact calculation all potentials are consistent with the data, boson exchange models leading to equivalent results as inversion potentials. Additionally, as a surprising result the cross section for $(p, p \gamma)$ Bremsstrahlung, which was assumed to be sensitive to the off-shell behavior of the $t$-matrix, can not distinguish between the original Bonn-B potential (dashed line) and the on-shell equivalent but off-shell different inversion potential (dotted) obtained from Bonn-B model phase shifts! Consequently, nothing can be learned about the off-shell properties of the NN scattering amplitude from $(p$, $p \gamma)$ Bremsstrahlung.

\section{B. Triton Binding Energies}

Analogue to $(p, p \gamma)$ Bremsstrahlung calculations, a number of potentials were applied to compute binding energies of ${ }^{3} \mathrm{H}$ [10]. As representative examples we consider model and inversion potentials for the Paris and Bonn-B potentials. The results follow the well-known correlation between the deuteron $D$-state probability and the binding energy $E_{B}\left({ }^{3} \mathrm{H}\right)$ which is shown in Figure 3 for various models. All of the model predictions underestimate the experimental value of $8.48 \mathrm{MeV}$, a circumstance which will be discussed below. For the Bonn-B and Paris potential the exact values are listed in Table 11$]$. Note that for the Paris original and inversion potential the triton binding energies are the same whereas for BonnB a significant difference arises. The reason can be seen from an argument pointed out by 
Machleidt [13]. The on-shell $t$-matrix is related to the central and tensor potential by the approximate relation

$$
t(k, k) \approx V_{C}(k, k)-\int d^{3} \vec{k}^{\prime} V_{T}\left(k, k^{\prime}\right) \frac{M}{k^{\prime 2}-k^{2}-i \epsilon} V_{T}\left(k^{\prime}, k\right) .
$$

Thus, for the genuine Bonn-B and the Bonn-B inversion potential, which are on-shell equivalent, the sum of Born term and integral term in (4.2) is equal, while the respective terms themselves may be different. The deuteron $D$-state probability on the other hand is dominantly determined by the tensor potential

$$
P_{D} \approx \int_{0}^{\infty} \int_{0}^{\infty} k^{2} d k k^{\prime 2} d k^{\prime} \frac{V_{T}\left(k, k^{\prime}\right)}{-E_{B}\left({ }^{2} \mathrm{H}\right)-\frac{k^{2}}{M}} \Psi_{0}(k)
$$

where $\Psi_{0}(q)$ is the deuteron $S$-wave. Thus, two on-shell equivalent potentials may indeed lead to different $D$-state probabilities and triton binding energies due to differences in the tensor potential parts. Obviously, this is the case for the Bonn-B original and inversion potential. This can be understood from the fact that inversion potentials are first of all local functions of $r$ in coordinate space since the scattering phase shifts as functions of $k$ build a one-dimensional manifold (see Section [II). However, the microscopic boson exchange potentials, which are naturally formulated in momentum space, are not necessarily local and this deviation may account for the differences in the tensor potential. Comparing the results for the Bonn-B and Paris potentials, one finds that the non-locality of the Paris potential obviously can be well represented by an on-shell equivalent local potential, whereas the non-locality of the Bonn-B potential produces a significant decrement of the $D$-state probability. This arises from the fact that the tensor force in the Paris potential is local, whereas the tensor potential in the Bonn-B potential is not. Thus, the nonlocality of the other potential contributions essentially play no role in the calculation of the triton binding energy.

After all, the calculation of triton binding energies shows some sensitivity to the non-local structure of potentials. Unfortunately, there is an ongoing discussion about the influence of three-body forces and relativistic corrections on the triton binding energy so that none of the above results can be favored. Further work on this field seems desirable. 


\section{Nucleon-Nucleus Scattering}

Embedded in an in-medium full-folding optical potential model various boson exchange and inversion potentials have been applied to calculate observables of nucleon-nucleus scattering [11]. In Figure团 we show the results obtained for the differential cross section and analyzing power for ${ }^{40} \mathrm{Ca}(p, p)$ scattering at $500 \mathrm{MeV}$ using the Paris potential together with inversion potentials from Paris model phase shifts and phases from the Arndt SM94 PWA [14]. The conclusions are twofold: As in the case of $(p, p \gamma)$ Bremsstrahlung, the genuine Paris and Paris inversion potential results can not be distinguished by the experiment and thus no off-shell sensitivity can be found in the analysis of elastic nucleon-nucleus scattering. Additionally, a new effect occurs comparing the inversion potentials from SM94 phase shifts and the Paris potential. The SM94 inversion potential, which by construction fits the elastic NN data much better than the Paris potential, yields significant better results for the cross section and the analyzing power. Conclusively, an improvement in the description of on-shell data also yields better results in nucleon-nucleus scattering.

\section{SUMMARY AND OUTLOOK}

As described in Section [1] and [II, quantum inversion and boson exchange potentials rest on entirely different footings. Whereas the latter are derived from some microscopic model, as the model of solitary mesons, leading to a momentum space potential with in general - nonlocal behavior, the inversion potentials are obtained model-independently from the Gel'fand-Levitan or Marchenko algorithms. Since elastic NN scattering data enter the inversion potentials via the phase shifts $\delta_{\ell}(k)$ as a one-dimensional manifold, inversion potentials are local, energy-independent functions in coordinate space. This fundamental difference could be expected to produce significant deviations in the description of processes where the off-shell part of the $t$-matrix, which does not enter the phase shifts, contributes.

Surprisingly, the application of boson exchange and inversion potentials in the calculation 
of $(p, p \gamma)$ Bremsstrahlung cross sections and nucleon-nucleus scattering observables produces equivalent results for the boson exchange potentials an their local counterparts. Additionally, in the case of nucleon-nucleus scattering it turned out that an improvement of the description of on-shell data also yields better results for off-shell observables.

The only difference in the comparison of boson exchange versus quantum inversion potentials occurred in the calculation of triton binding energies. It turned out that the usage of local potentials enhances the $D$-state probability with respect to their non-local on-shell equivalents, leading to a lower triton binding energy. This effect could be traced back to differences in the tensor part of the potential which can differ for local and non-local potentials. Unfortunately, neither boson exchange nor quantum inversion potentials can be favored in this context due to the persistent uncertainty concerning the effect of three-body forces and relativistic corrections on the triton binding energy.

To our disappointment, the study of the above reactions thus is not suitable to put boson exchange potentials to a comparative test. Therefore, as a future prospect, the application of boson exchange and inversion potentials in meson-nucleon and meson-meson scattering seems promising to test the concept of quantum inversion as well as the applicability of NN potential models in a wider range of hadron-hadron interactions. In this context, the OSBEP model can be expected to show interesting results. In contrast to conventional boson exchange potentials, which use different form factors in nucleon-nucleon and meson-nucleon interactions, the concept of proper normalization in OSBEP remains unchanged. Thus, the OSBEP model may be able to describe both interactions consistently with a very small number of parameters.

\section{ACKNOWLEDGMENTS}

Supported in part by FZ Jülich, COSY Collaboration, Grant Nr. 41126865. 


\section{REFERENCES}

[1] M. M. Nagels, T. A. Rijken, and J. J. de Swart, Phys. Rev. D 17, 768 (1978); M. Lacombe, B. Loiseau, J. M. Richard, R. Vinh Mau, J. Côté, P. Pirès, and R. de Tourreil, Phys. Rev. C 21, 861 (1980); R. Machleidt, K. Holinde, and Ch. Elster, Physics Reports 149, 1 (1987).

[2] T. H. R. Skyrme, Nucl. Phys. 31, 556 (1962); S. Weinberg, Phys. Rev. Lett. 18, 188 (1967); J. Wambach, in Quantum Inversion Theory and Applications, edited by H. V. von Geramb (Lecture Notes in Physics, Springer, New York, 1994); C. Ordóñez, L. Ray, and U. van Kolck, Phys. Rev. Lett. 72, 1982 (1994); C. M. Shakin, Wei-Dong Sun, and J. Szweda, Phys. Rev. C 52, 3353 (1995).

[3] L. Jäde and H. V. von Geramb, LANL e-print archive nucl-th/9604002, submitted to Phys. Rev. C.

[4] R. Machleidt, Adv. in Nucl. Phys. 19, 189 (1989).

[5] H. Kohlhoff and H. V. von Geramb, in Quantum Inversion Theory and Applications, Proceedings of the 109th W.E. Heraeus Seminar, Bad Honnef 1993, edited by H. V. von Geramb (Lecture Notes in Physics, Springer, New York, 1994); M. Sander, C. Beck, B. C. Schröder, H.-B. Pyo, H. Becker, J. Burrows, H. V. von Geramb, Y. Wu, and S. Ishikawa, in Conference Proceedings, Physics with GeV-Particle Beams (World Scientific, Singapore 1995).

[6] P. B. Burt, Quantum Mechanics and Nonlinear Waves (Harwood Academic, New York, 1981).

[7] C. Itzykson and J. B. Zuber, Quantum Field Theory (Mc. Graw-Hill, New York 1980).

[8] L. Jäde and H. V. von Geramb, LANL e-print server, nucl-th/9510061 (1995).

[9] M. Jetter and H. V. von Geramb, Phys. Rev. C 49, 1832 (1994). 
[10] B. F. Gibson, H. Kohlhoff, and H. V. von Geramb, Phys. Rev. C 51, R465 (1995).

[11] H. F. Arellano, F. A. Brieva, M. Sander, and H. V. von Geramb, to appear in Phys. Rev. C 54 (1996).

[12] V. Stoks and J. J. de Swart, Phys. Rev. C 48, 792 (1993).

[13] R. Machleidt and G. Q. Li, LANL e-print server, nucl-th/9301019 (1993).

[14] R. A. Arndt, I. I. Strakovsky and R. L. Workman, Phys. Rev. C 50, 2731 (1994). 


\section{TABLES}

TABLE I. OSBEP parameters

\begin{tabular}{|c|c|c|c|c|c|c|c|}
\hline & $\pi$ & $\eta$ & $\rho$ & $\omega$ & $\sigma_{0}$ & $\sigma_{1}$ & $\delta$ \\
\hline$\overline{S^{P}}$ & $0^{-}$ & $0^{-}$ & $1^{-}$ & $1^{-}$ & $0^{+}$ & $0^{+}$ & $0^{+}$ \\
\hline$\frac{g_{\beta}^{2}}{\Delta \pi}$ & 13.7 & 1.3985 & 1.1398 & 18.709 & 14.147 & 7.8389 & 1.3688 \\
\hline \multicolumn{4}{|c|}{$\alpha_{\pi}=0.428321$} & \multicolumn{4}{|c|}{$f_{\rho} / g_{\rho}=4.422$} \\
\hline
\end{tabular}

TABLE II. Deuteron Properties

\begin{tabular}{llll}
\hline \hline & Bonn-B $^{\mathrm{a}}$ & OSBEP & Exp. \\
\hline$E_{B}(\mathrm{MeV})$ & 2.2246 & 2.22459 & $2.22458900(22)$ \\
$\mu_{d}\left(\mathrm{fm}^{2}\right)$ & $0.8514^{\mathrm{b}}$ & $0.8532^{\mathrm{b}}$ & $0.857406(1)$ \\
$Q_{d}\left(\mathrm{fm}^{-1 / 2}\right)$ & $0.2783^{\mathrm{b}}$ & $0.2670^{\mathrm{b}}$ & $0.2859(3)$ \\
$A_{S}$ & 0.8792 & $0.8802(20)$ \\
$D / S$ & 0.8860 & 0.0256 & $0.0256(4)$ \\
$r_{R M S}(\mathrm{fm})$ & 0.0264 & 1.9539 & $1.9627(38)$ \\
$P_{D}(\%)$ & 1.9688 & 4.6528 & - \\
\hline \hline
\end{tabular}

${ }^{\mathrm{a} D a t a}$ from [州]

${ }^{\mathrm{b}}$ Meson exchange current contributions not included

TABLE III. Deuteron $D$-State Probabilities and Triton Binding Energies

\begin{tabular}{lllll}
\hline \hline & Paris & Paris (inv.) & Bonn-B & Bonn-B (inv.) \\
\hline$P_{D}$ & $5.77 \%$ & $5.69 \%$ & $4.99 \%$ & $5.81 \%$ \\
$E_{B}\left({ }^{3} \mathrm{H}\right)$ & $7.47 \mathrm{MeV}$ & $7.47 \mathrm{MeV}$ & $8.14 \mathrm{MeV}$ & $7.84 \mathrm{MeV}$ \\
\hline \hline
\end{tabular}




\section{FIGURES}
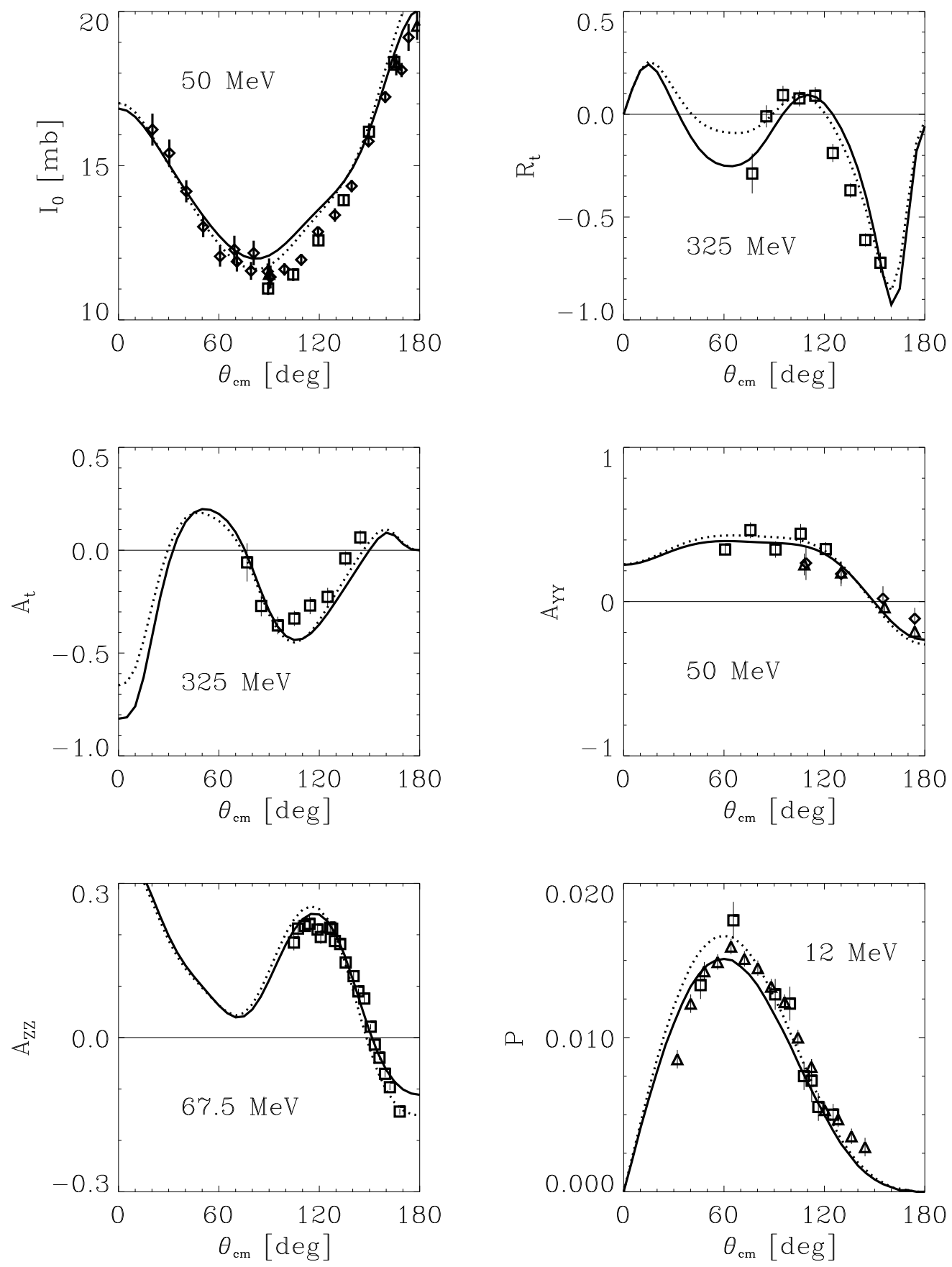

FIG. 1. Observables of $n p$ scattering. Kinetic laboratory energy is denoted, experimental data are taken from the VPI-SAID program. We show theoretical predictions from OSBEP (full) and Bonn-B (dotted). 

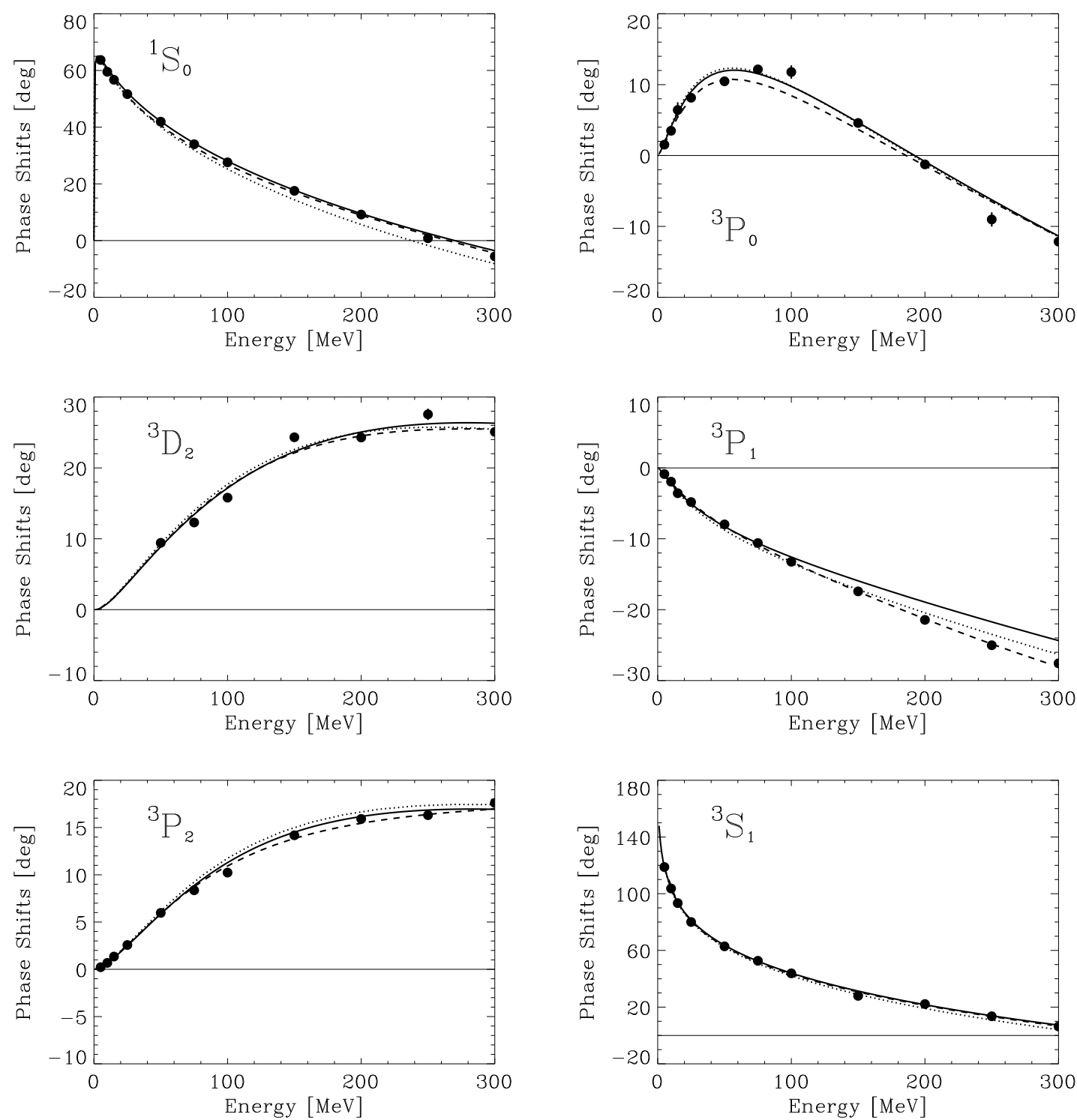

FIG. 2. Selected $n p$ phase shifts: Arndt SM95 (circles), Nijmegen PWA (dashed), Bonn-B (dotted) and OSBEP (full). 

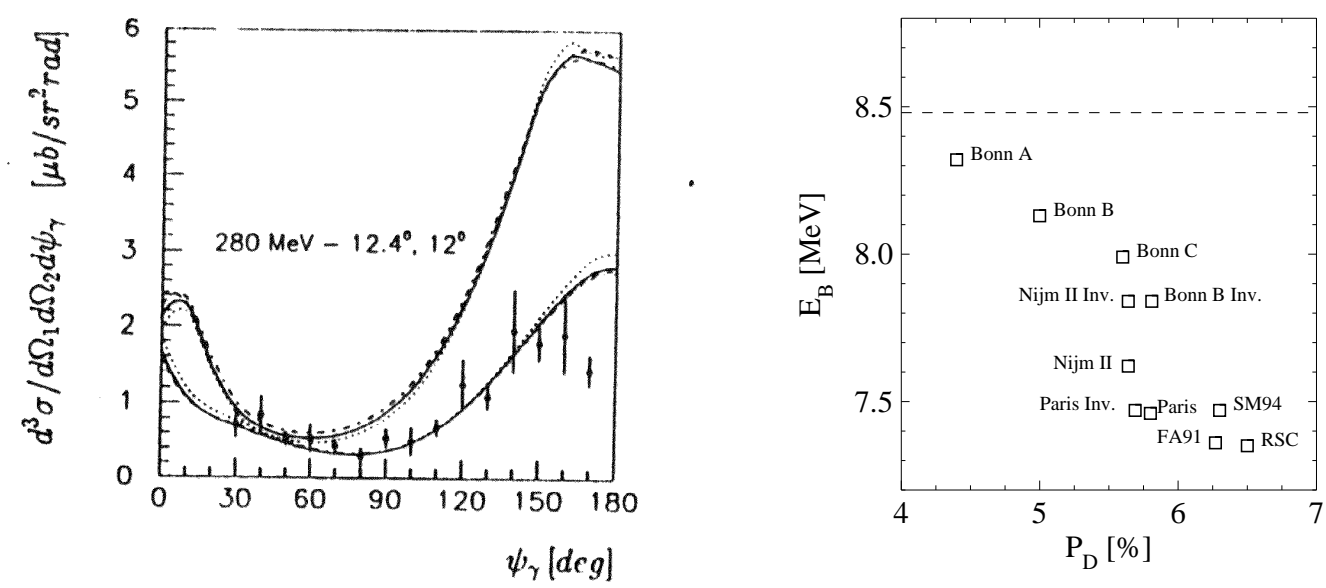

FIG. 3. Left figure: Theoretical $(p, p \gamma)$ cross sections from various boson exchange and inversion potentials. The lower curves show the results for an exact calculation whereas the upper curves represent an on-shell approximation. The lines are Nijmegen PWA inversion (solid), Paris (dash-dotted), Bonn-B (dashed) and an inversion potential from Bonn-B phase shifts (dotted). Right figure: Triton binding energy versus deuteron $D$-state probability for various potentials. Note the large differences in both entities for the genuine and inversion Bonn-B potential, whereas Paris original and Paris inversion show similar results.
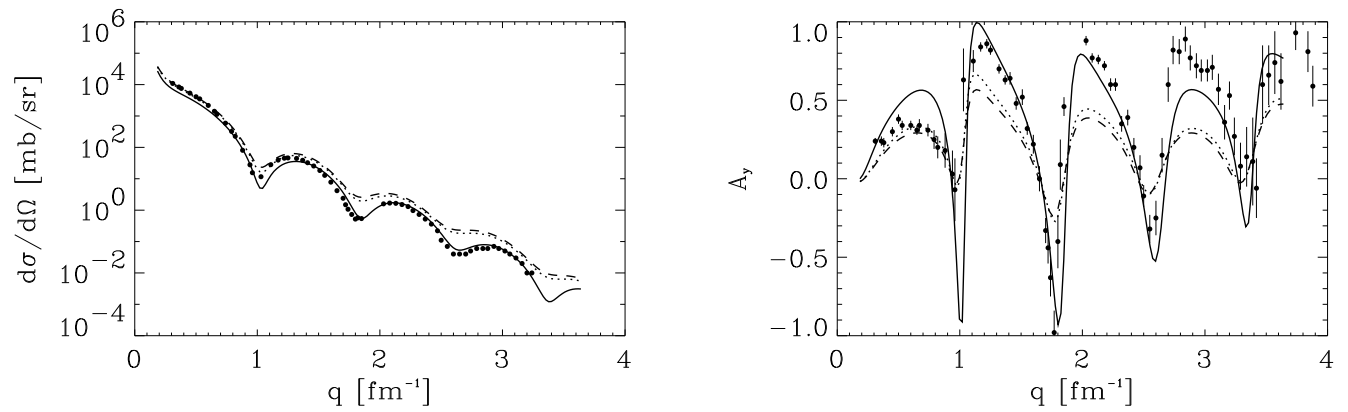

FIG. 4. Differential cross section (left) and analyzing power (right) for ${ }^{40} \mathrm{Ca}(p, p)$ at $500 \mathrm{MeV}$. We show results for SM94 inversion (solid), genuine Paris potential (dotted) and Paris inversion (dashed). 